\title{
Field Distribution of Epidural Electrical Stimulation
}

\author{
Xiaobo Xie ${ }^{1, \#}$, HY Cui ${ }^{1, \#}$, Shengpu $\mathrm{Xu}^{1}$, Yong $\mathrm{Hu}^{1,2, *}$
}

1. Institute of Biomedical Engineering, Chinese Academy of Medical Sciences and Peking Union Medical College, People's Republic of China

2. Dept of Orthopaedics and Traumatology. The University of Hong Kon, Pokfulam, Hong Kong

${ }^{\#}$ These authors contributed equally to this work

${ }^{*}$ Corresponding author:

Dr Yong $\mathrm{Hu}$

Institute of Biomedical Engineering, Chinese Academy of Medical Science and Peking Union Medical College, People's Republic of China

E-mail: yhud@hku.hk

Acknowledgements: This work was supported in part by the Hong Kong Research Grants Council (GRF HKU767511E), and the Tianjin Key Project Grant (No.11ZCKFSY01600). 


\section{Abstract}

Epidural electrical stimulation has been applied in clinics for many years. However, it is still a concern about possible injury to the spinal nerve. This study investigated electrical field and current density distribution during direct epidural electrical stimulation. Field distribution models were theoretically deduced, while the distribution of potentials and current were analyzed. The current density presented an increase of $70-80 \%$, with one peak value ranged from $-85^{\circ}$ to $85^{\circ}$ between the two stimulated poles. The effect of direct epidural electrical stimulation is mainly on local tissue surrounding the electrodes, which concentrates around two simulated positions.

Key words: spinal cord; epidural electrical stimulation; electrical field distribution; current density distribution 


\section{INTRODUCTION}

In 1981, Tamaki et al. achieved spinal cord evoked potential (SCEP) by applying the direct spinal cord stimulation method, thereby eliminating effects from surrounding nerves and collecting evoked potential signals with better signal to noise ratio (SNR) and higher amplitude. This technology has since been applied during intraoperative spinal cord monitoring and many other clinical therapies, such as repair of spinal cord injury, convulsion treatment, and asthenia [1-4]. However, direct spinal cord stimulation takes place near the spinal cord and spinal nerves [5], which could result in injury to the spinal cord or other nerves. In addition, the latency of evoked potential stimulated by direct spinal cord stimulation is shorter than other transcranial EPs; it is difficult to eliminate stimulus artifacts from electrical stimulation using the filter method, so stimulation intensity is limited[6]. Although clinical and animal experiments have illustrated that direct spinal cord electrical stimulation can effectively excite spinal nerves [7], several problems still remain. For example, it remains to be shown whether electrode placement affects nerve stimulation, or whether direct electrical stimulation results in partial or whole spinal nerve excitement $[8,9]$. Further studies on these issues will provide further knowledge for clinical monitoring of direct spinal cord stimulation.

At present, studies have addressed mathematical models of potential field and current density field distribution during electrical stimulation, and some have simulated these models using the finite element method [10-17]. Unfortunately, the finite element method cannot prodict the integral distribution, so it is difficult to identify boundary conditions due to anatomical complexities of the human body.

The current study investigated the rule of electrical field and current density distributions within the spinal cord during direct epidural electrical stimulation, constructed an approximate mathematical model, and deduced an analytical solution to the model. Subsequently, the mechanisms of epidural electrical stimulation on the spinal nerves were analyzed to provide an explanation for the integral distribution rule of electrical fields. These results provide theoretical instruction for the application of spinal cord evoked potential during epidural electrical stimulation. 


\section{METHOD}

To apply direct spinal cord electrical stimulation through electrodes located at the epidural space of the spine, it is necessary to simplify the complex structures of the spinal cord and dura matter. The spinal cord resides in the vertebral canal, wrapped by dura matter. A cross section of the lower cervical vertebra, thoracic vertebra, and lumbar region is almost cylindrical. The entire spinal cord is approximately $40-45 \mathrm{~cm}$ long. A transverse section is shown in Figure 1; the gray matter is in the centre like an "H" or a butterfly, and the white matter surrounds this [18].

Because the difference between conduction characteristics of white and gray matter is small, the inner region of the spinal cord can be considered a cylindrical dielectric. In clinical application, the stimulating electrodes are usually placed between the upper thoracic vertebra and lumbar. At this point the spinal cord and dura matter form an outstretching cylinder; the inner spinal cord consists of nerve fibers and the outer region contains dura matter. A direct current $I$ was applied to the dura matter surface. The semidiameter of electrodes is smaller than for the spine, so the interface between electrodes and dura matter was considered to be a quadrate surface, with length $c_{1}$ and width $c_{2}$, where $c_{1}$ was the width of electrode wires and $c_{2}$ was the diameter of electrode, as shown in Figure 2.

In Figure 3, $a$ depicts the semidiameter of the spinal cord, and $b$ was the distance between the dura matter surface and the center of the spinal cord. A point current source $I_{\mathrm{s}}$, located on the interface between electrode and dura matter surface, is placed at $\left(b, 0, z_{0}\right)$ outside the cylinder, where $0 \leq z_{0} \leq c_{1}$. We assumed the horizontal angle of $\left(b, 0, z_{0}\right)$ was $\theta_{0}=0$, the axial distance was $z=z_{0}$, and $(\rho, \theta, z)$ was a random point in the spinal cord.

\section{A. Bessel Function Deduction}

It was assumed that tissues from the spinal cord and dura matter are isotropic, or 
homogenous dielectric, and that the cylinder boundary effect was negligible. $\sigma$ was the electrical conductivity within relevant space. So, $\sigma=\sigma_{i}$ was the electrical conductivity within the spinal cord and $\sigma=\sigma_{o}$ was the electrical conductivity within the dura matter. On the basis of electromagnetic field theory, the potentials evoked by a point electrical source in the spinal cord and dura matter can be defined as:

$$
\phi=\frac{I_{s}}{4 \pi \sigma R}
$$

where $\sigma$ was electrical conductivity within a relevant space, and $R$ was the distance between a point electrical source and a random point $(\rho, \theta, z)$ in the spine.

According to Figure 4, if the origin of two-dimensional cylindrical coordinates is moved along the $x$ axes by a distance of $b$, then distance $r$ between the new origin and a point $(\rho, \theta)$ on the old two-dimensional coordinates can be defined as:

$$
r^{2}=b^{2}+\rho^{2}-2 b \rho \cos \theta
$$

Subsequently, $R$ can be calculated as:

$$
R=\sqrt{b^{2}+\rho^{2}-2 b \rho \cos \theta+\left(z-z_{0}\right)^{2}}
$$

i.e. $\quad \frac{1}{R}=\frac{1}{\sqrt{r^{2}+\left(z-z_{0}\right)^{2}}}$

According to geometry theory[19]: if two right-angled lines of a right triangle are $z$ and $r$, respectively, then the hypotenuse can be defined as the Fourier integral of the modified Bessel Function, as follows:

$$
\begin{gathered}
\frac{1}{\sqrt{r^{2}+\left(z-z_{0}\right)^{2}}}=\frac{1}{\pi} \int_{-\infty}^{+\infty} K_{0}(k r) \mathrm{e}^{j k\left(z-z_{0}\right)} \mathrm{d} k \\
=\mathrm{e}^{-z_{0}} \frac{1}{\pi} \int_{-\infty}^{+\infty} K_{0}(k r) \mathrm{e}^{j k z} \mathrm{~d} k
\end{gathered}
$$

The weighted sum equation of the modified Zeroth-order Bessel Function of the second kind is as follows:

$$
K_{0}(k r)=K_{0}(k b) I_{0}(k \rho)+2 \sum_{n=1}^{\infty} K_{n}(k b) I_{n}(k \rho) \cos n \theta
$$


Because the Cosine Function is Cosine Function at $\theta=0$, equation (6) can be reduced to (7):

$$
K_{0}(k r)=\sum_{n=-\infty}^{\infty} K_{n}(k b) I_{n}(k \rho) \cos n \theta
$$

Where $I_{n}(k \rho)$ and $K_{n}(k b)$ are the first kind and second kind of the modified $n$ th-order Bessel Function, respectively.

According to equations (4), (5), and (7), changes in the order of weight sum and integral, as well as distance $R$ between point electrical source and a random point $(\rho, \theta, z)$ can be defined by:

$$
\frac{1}{R}=\mathrm{e}^{-z_{0}} \frac{1}{\pi} \sum_{n=-\infty}^{+\infty} \int_{-\infty}^{+\infty} K_{n}(k b) I_{n}(k \rho) \cos n \theta \mathrm{e}^{j k z} \mathrm{~d} k
$$

Therefore, equation (1) could be reduced to the following equation:

$$
\phi=\mathrm{e}^{-z_{0}} \frac{I_{s}}{4 \pi^{2} \sigma} \sum_{n=-\infty}^{+\infty} \int_{-\infty}^{+\infty} K_{n}(k b) I_{n}(k \rho) \cos n \theta \mathrm{e}^{j k z} \mathrm{~d} k
$$

Equation (9) is the potential in an isotropic, homogenous dielectric during point electrical source stimulation. For the idealized model used in this study, electrical conductivities within the spinal cord and dura matter varied. Therefore, conductivity was not discrete, and there was induced charge on the interface between the spinal cord and dura matter, which induced an electrical field.

\section{B. Construction of the Field Distribution models}

Assume that potentials in the spinal cord and dura matter are:

$$
\begin{aligned}
& \phi_{\text {in }}=\phi_{\text {ins }}+\phi_{\text {applied }} \\
& \phi_{\text {out }}=\phi_{\text {outs }}+\phi_{\text {applied }}
\end{aligned}
$$

Where $\phi_{\text {in }}$ was the spinal cord potential, and $\phi_{\text {out }}$ is the dura matter potential, $\phi_{\text {applied }}$ was the potential produced by the point electrical source, and $\phi_{\text {ins }}$ and $\phi_{\text {outs }}$ were induced potential in the spinal cord and dura matter, respectively.

In the dura matter, the induced electrical field follows the Laplacian Equation[19], 
as follows:

$$
\frac{\partial^{2} \phi_{\text {outs }}}{\partial \rho^{2}}+\frac{1}{\rho} \frac{\partial \phi_{\text {outs }}}{\partial \rho}+\frac{1}{\rho^{2}} \frac{\partial^{2} \phi_{\text {outs }}}{\partial \theta^{2}}+\frac{\partial^{2} \phi_{\text {outs }}}{\partial \mathrm{z}^{2}}=0
$$

To solve this partial difference equation using the method of Separation of Variables in cylindrical coordinates[18], it could be displayed as the product of the three parameters: $\rho, \theta$, and $\mathrm{z}$. Therefore, the solution was:

$$
\left(a_{n} \sin (n \theta)+b_{n} \cos (n \theta)\right)\left(c_{n} I_{n}(k \rho)+d_{n} K_{n}(k \rho)\right)\left(e_{n} \mathrm{e}^{j k z}+f_{n} \mathrm{e}^{-j k z}\right)
$$

where $I_{n}(k \rho)$ and $K_{n}(k \rho)$ were the first kind and second kind of the modified $n$ th-order Bessel Function, respectively. According to symmetry, the potential was the same at the positive and negative angles of $\theta$. Therefore, $a_{n}$ should be 0 .

\section{Potential in the dura matter}

When $\rho \rightarrow \infty, I_{n}(k \rho)$ is $\log$ infinite, equation (11) becomes infinite. Therefore, only the second kind of Bessel Function $K_{n}(k \rho)$ was applicable to the area outside the smaller cylinder. In other words, $c_{n}=0$ when $\rho \rightarrow \infty$. According to relation $K_{n}(x)=K_{-n}(x)$, the sum of $n$ from two sides and the integral for $k$ was displayed from $-\infty$ to $+\infty$, and the potential in dura matter was produced by induced charge:

$$
\begin{aligned}
\phi_{\text {outs }}(\rho, \theta, z) & =\sum_{n=-\infty}^{+\infty} \int_{-\infty}^{+\infty} D_{n}(k) K_{n}(k \rho) \cos n \theta \mathrm{e}^{-j k\left(z-z_{0}\right)} \mathrm{d} k \\
& =\mathrm{e}^{z_{0}} \sum_{n=-\infty}^{+\infty} \int_{-\infty}^{+\infty} D_{n}(k) K_{n}(k \rho) \cos n \theta \mathrm{e}^{-j k z} \mathrm{~d} k
\end{aligned}
$$

Finally, for a point electrical source with a current density $I_{s}$, the total potential in dura matter was:

$$
\phi_{\text {out }}(\rho, \theta, z)=\mathrm{e}^{z_{0}} \sum_{n=-\infty}^{+\infty} \int_{-\infty}^{+\infty} D_{n}(k) K_{n}(k \rho) \cos n \theta \mathrm{e}^{-j k z} \mathrm{~d} k+\frac{I_{s}}{4 \pi \sigma_{0} R}
$$

where $\sigma_{o}$ was the electrical conductivity in dura matter, and the coefficient $D_{n}(k)$ was determined by boundary conditions. 


\section{Potential in the spinal cord}

Because the spinal cord has a passive field, the applied field and induced field for a spinal cord point were combined, and the combination follows the Laplacian Equation:

$$
\frac{\partial^{2} \phi_{\text {in }}}{\partial \rho^{2}}+\frac{1}{\rho} \frac{\partial \phi_{\text {in }}}{\partial \rho}+\frac{1}{\rho^{2}} \frac{\partial^{2} \phi_{\text {in }}}{\partial \theta^{2}}+\frac{\partial^{2} \phi_{\text {in }}}{\partial z^{2}}=0
$$

Equation (11) could be subsequently calculated. Because $K_{n}(k \rho) \rightarrow \infty$ when $\rho \rightarrow 0$, then $d_{n}=0$. In addition, $I_{n}(x)=I_{-n}(x)$ was used to calculate potential in spinal cord as:

$$
\begin{aligned}
\phi_{\text {in }}(\rho, \theta, z) & =\sum_{n=-\infty}^{+\infty} \int_{-\infty}^{+\infty} C_{n}(k) I_{n}(k \rho) \cos n \theta \mathrm{e}^{-j k\left(z-z_{0}\right)} \mathrm{d} k \\
= & \mathrm{e}^{z_{0}} \sum_{n=-\infty}^{+\infty} \int_{-\infty}^{+\infty} C_{n}(k) I_{n}(k \rho) \cos n \theta \mathrm{e}^{-j k z} \mathrm{~d} k
\end{aligned}
$$

The next step was to determine coefficients $C_{n}(k)$ and $D_{n}(k)$ using proper boundary conditions. In this model, the difference between the electrical conductivities of dura matter and spinal cord was very small, and therefore the resistance on the interface could be neglected. Therefore, the boundary conditions of the cylinder interface were: (a) potential was continuous; (b) radial current density was continuous, and could be expressed as:

$$
\begin{gathered}
\phi_{\text {in }}(\rho, \theta, z)_{\rho=a}=\phi_{\text {out }}(\rho, \theta, z)_{\rho=a} \\
\sigma_{o}\left(\frac{\partial \phi_{\text {out }}}{\partial \rho}\right)_{\rho=a}=\sigma_{i}\left(\frac{\partial \phi_{\text {in }}}{\partial \rho}\right)_{\rho=a}
\end{gathered}
$$

where $\sigma_{i}$ was the electrical conductivity in the spinal cord and $\sigma_{o}$ was the electrical conductivity in the dura matter.

Replacing the relevant parts in equations (13) and (15) with the above boundary conditions, the coefficients $C_{n}(k)$ and $D_{n}(k)$ could be deduced as follows: 


$$
\begin{gathered}
C_{n}(k)=\frac{I_{s} K_{n}(k b)\left[I_{n}(k a) K_{n}{ }^{\mathrm{n}}(k a)-I_{n}{ }^{\mathrm{n}}(k a) K_{n}(k a)\right]}{4 \pi^{2}\left[\sigma_{o} I_{n}(k a) K_{n}{ }^{\mathrm{n}}(k a)-\sigma_{i} I_{n}{ }^{\mathrm{n}}(k a) K_{n}(k a)\right]} \\
D_{n}(k)=\frac{I_{s}\left(\frac{\sigma_{i}}{\sigma_{o}}-1\right) K_{n}(k b) I_{n}(k a)}{4 \pi^{2}\left[\sigma_{o} I_{n}(k a) K_{n}{ }^{\mathrm{n}}(k a)-\sigma_{i} I_{n}{ }^{\mathrm{n}}(k a) K_{n}(k a)\right]}
\end{gathered}
$$

Potentials in the dura matter and spinal cord could be calculated by putting equations (18) and (19) into equation (13) and (15).

Thus far, we obtained potential field distribution from dura matter and spinal cord during direct epidural electrical stimulation by point electrical source. According to the addition theory of electrical field, integral equations (9), (13), and (15) were within the area of the stimulation electrode. Deduction of field distribution during interface stimulated:

$$
\begin{aligned}
\left.\phi\right|_{s} & =\int_{s} \phi=\int_{s}\left[\mathrm{e}^{-z_{0}} \frac{I_{s}}{4 \pi^{2} \sigma} \sum_{n=-\infty}^{+\infty} \int_{-\infty}^{+\infty} K_{n}(k b) I_{n}(k \rho) \cos n \theta \mathrm{e}^{j k z} \mathrm{~d} k\right] \\
& =\int_{s} \mathrm{e}^{-z_{0}} \mathrm{~d} s\left[\frac{I_{s}}{4 \pi^{2} \sigma} \sum_{n=-\infty}^{+\infty} \int_{-\infty}^{+\infty} K_{n}(k b) I_{n}(k \rho) \cos n \theta \mathrm{e}^{j k z} \mathrm{~d} k\right] \\
& =\frac{\left[-C_{2}\left(\mathrm{e}^{-c_{1}}-1\right)\right] I_{s}}{4 \pi^{2} \sigma}\left[\sum_{n=-\infty}^{+\infty} \int_{-\infty}^{+\infty} K_{n}(k b) I_{n}(k \rho) \cos n \theta \mathrm{e}^{j k z} \mathrm{~d} k\right] \\
\left.\phi_{\text {out }}\right|_{s} & =\int_{s} \phi_{\text {out }}=\int_{s}\left[\mathrm{e}^{z_{0}} \sum_{n=-\infty}^{+\infty} \int_{-\infty}^{+\infty} D_{n}(k) K_{n}(k \rho) \cos n \theta \mathrm{e}^{-j k z} \mathrm{~d} k\right]+\frac{I_{s}}{4 \pi \sigma_{0} R} \\
& =C_{2}\left(\mathrm{e}^{c_{1}}-1\right)\left[\sum_{n=-\infty}^{+\infty} \int_{-\infty}^{+\infty} D_{n}(k) K_{n}(k \rho) \cos n \theta \mathrm{e}^{-j k z} \mathrm{~d} k\right]+\frac{I_{s}}{4 \pi \sigma_{0} R} \\
\left.\phi_{\text {in }}\right|_{s} & =\int_{s} \phi_{\text {in }}=\int_{s}\left[\mathrm{e}^{z_{0}} \sum_{n=-\infty}^{+\infty} \int_{-\infty}^{+\infty} C_{n}(k) I_{n}(k \rho) \cos n \theta \mathrm{e}^{-j k z} \mathrm{~d} k\right] \\
& =C_{2}\left(\mathrm{e}^{c_{1}}-1\right)\left[\sum_{n=-\infty}^{+\infty} \int_{-\infty}^{+\infty} C_{n}(k) I_{n}(k \rho) \cos n \theta \mathrm{e}^{-j k z} \mathrm{~d} k\right]
\end{aligned}
$$

Finally, according to the differential form of Ohm's law below:

$$
\begin{aligned}
& \vec{E}(r, z)=-\vec{\nabla} \phi(r, z) \\
& \vec{J}(r, z)=\sigma \vec{E}(r, z)
\end{aligned}
$$

we calculated current density distribution in the dura matter and spinal cord. To accomplish computer simulation, it is necessary to discretize the above equations. 


\section{Model discretization}

First, the integral of $k$ was discretized. Here it was assumed that $k$ and $n$ were the spatial frequency along the $Z$ and $\theta$ axis, respectively, $Z$ and $S$ were the spatial sampling interval along the $\mathrm{z}$ and $\theta$ axis, respectively, and $2 \mathrm{M}$ and $2 \mathrm{~N}$ were the sampling hits along the $z$ and $\theta$ axis, respectively. This resulted in $k=$ $\pi / M Z$ and $n=\pi / N S$.

Second, $\cos n \theta$ was transformed into a complex exponential form. For a random integer $l$ and $q$, where $-M \leq l \leq M-1$ and $-N \leq q \leq N-1$, the Fast Fourier Transform of equations (20), (21), and (22) should be performed. They could be formulated as follows, respectively:

$$
\left.\phi(\rho, q S, I Z)\right|_{s}=\frac{\left[-C_{2}\left(\mathrm{e}^{-c_{1}}-1\right)\right] I_{s}}{4 \pi^{2} \sigma} \frac{\pi}{M Z} \sum_{n=1}^{N-1} \sum_{m=1}^{M-1}\left[K_{n}\left(\frac{\pi m}{M Z} b\right) I_{n}\left(\frac{\pi m}{M Z} \rho\right)\right] \mathrm{e}^{-j \pi m l / M} \mathrm{e}^{-j \pi n q / N}
$$

$$
\begin{aligned}
\left.\phi_{\text {out }}(\rho, q S, I Z)\right|_{s}= & {\left[c_{2}\left(\mathrm{e}^{c_{1}}-1\right)\right] \sum_{n=-N}^{N-1} \sum_{m=-M}^{M-1}\left[D_{n}\left(\frac{\pi m}{M Z}\right) K_{n}\left(\frac{\pi m}{M Z} \rho\right)\right] \mathrm{e}^{-j \pi m l / M} \mathrm{e}^{-j \pi n q / N} } \\
& +\left.\frac{I_{s}}{4 \pi \sigma_{o} R}\right|_{s}
\end{aligned}
$$

$$
\left.\phi_{\text {in }}(\rho, q S, l Z)\right|_{s}=\left[c_{2}\left(\mathrm{e}^{c_{1}}-1\right)\right] \sum_{n=-N}^{N-1} \sum_{m=-M}^{M-1}\left[C_{n}\left(\frac{\pi m}{M Z}\right) I_{n}\left(\frac{\pi m}{M Z} \rho\right)\right] \mathrm{e}^{-j \pi m l / M} \mathrm{e}^{-j \pi n q / N}
$$

where:

$$
\left.\frac{1}{4 \pi \sigma_{o} R}\right|_{s}=\frac{\left[-C_{2}\left(\mathrm{e}^{-c_{1}}-1\right)\right] I_{s}}{4 \pi^{2} \sigma_{o}} \frac{\pi}{M Z} \sum_{n=-N}^{N-1} \sum_{m=-M}^{M-1}\left[K_{n}\left(\frac{\pi m}{M Z} b\right) I_{n}\left(\frac{\pi m}{M Z} \rho\right)\right] \mathrm{e}^{-j \pi m l / M} \mathrm{e}^{-j \pi n q / N}
$$


MATLAB Software was used to simulate instantaneous field distribution within the dura matter and spinal cord during double electrode stimulation in contour maps. In the models, the length of the spinal cord segment was $5 \mathrm{~cm}$, and the longitudinal placement of the two electrodes was applied as depicted in Figure 2. The width of electrode wire $c_{1}$ was $0.05 \mathrm{~cm}$, the diameter of electrode $c_{2}$ was $0.075 \mathrm{~cm}$, the diameter of spinal cord $a$ was $1.5 \mathrm{~cm}$. The distance between the surface of dura matter and the center of spinal cord $b$ was $1.51 \mathrm{~cm}$, the negative and positive electrodes was longitudinally located at $(1.51 \mathrm{~cm}, 0,0.75 \mathrm{~cm})$ and $(1.51 \mathrm{~cm}, 0,-0.75$ $\mathrm{cm}$ ) respectively, electrical conductivity within dura matter $\sigma_{o}$ was $0.03 \mathrm{~S} / \mathrm{m}$, and electrical conductivity within spinal cord $\sigma_{i}$ was $0.083 \mathrm{~S} / \mathrm{m}[4,20]$, and a stimulating current intensity of $5 \mathrm{~mA}$ was used, resulting in $I_{s}=\frac{5}{C_{1} C_{2}} \mathrm{~mA}$ per $\mathrm{cm}^{2}$.

Based on the above assumptions, the three-dimensional figures were plotted to explain the distribution of potential and current density with different nerve tissues on cylindrical coordinates. Contour maps of potential and current density were also generated from these values and plotted. These figures helped to visualize the potential distribution and current density spread in each model. In this study, the plots were made along longitudinal $z$ and transverse $\theta$ axes.

\section{Three-dimensional plots and isopotential and isocurrent density contours}

In Figure 5, the distribution of potential within the dura matter tissue is shown. By comparing the two subplots, we could observe that the potential along $Z$ axis was narrower than along the $\theta$ axis. Therefore, we could conclude that stimulation spread primarily towards the inner spinal cord due to smaller electrical conductivity within the dura matter.

Figure 6-8 are three examples of potential and current density distributions within the spinal cord when $\rho=0.5 \mathrm{~cm}, \rho=1.0 \mathrm{~cm}$ and $\rho=1.5 \mathrm{~cm}$. In all models, there are two peak values of potentials located around the two stimulated sites, as shown on the 
subplots (b). When comparing the three figures, it could be observed that the potential increased proportionally while $\rho$ varied between $0.5-1.5 \mathrm{~cm}$. This illustrates that potential values in the outer spinal cord were greater that in the inner regions. In addition, potential distribution in all three cases was uniform at different $(z, \theta)$ points for a given $\rho$. The distribution increased with less distance from the stimulating source, which suggested that potential along transverse and longitudinal spinal cord regions is greatest at stimulated positions[21]. As shown in subplots (a) of Figure 6-8, for a given $\rho$, isopotential contours change only modestly with respect to distance away from the stimulated positions.

The distribution of current density within spinal cord varies from the potential field, as shown in Figure 9-11 with different $\rho$ values and only one peak value of current density. By comparing these three figures, it could be observed that current density increased when $\rho$ ranged from $0.5-1.5 \mathrm{~cm}$. In other words, current density in the outer spinal cord was more intensive than within the inner regions. Because the structure of the outer spinal cord transverse section primarily consists of white matter, these results suggest that if the stimulating intensity were limited to a certain range, the electrical stimulating effect on white matter around spinal cord would be far greater than in the central grey matter. Increased stimulating intensity would be required to stimulate the deeper conducting fibers.

For a given $\rho$ value, refer to Figure 9-11 along the axis. The current density was zero at the stimulated sites due to a combination of two vector fields produced by the stimulating electrodes. The current density was the most intense at the middle section of the two electrodes where $z=0$, which suggested that the most effected area was between the two electrodes and not at either position of the electrodes. In addition, the contour was symmetrical along $z=0$ and the lines were closer from the outer regions to the inner regions. All models displayed a decrease of $70-80 \%$ in current density from the peak value to the outer regions, which demonstrated that current density varied faster when it was closer to the center. 
Refer to Figure 9-11 along the $\theta$ axis. For a given $\rho$ value, the peak value was approximately $\theta=0$ and ranged from $-85^{\circ}$ to $85^{\circ}$, which demonstrated that the peak value was mostly around the line along the two electrode lines that ranged from $-85.98^{\circ}$ to $85.98^{\circ}$. The current density variations at other areas were milder. These results showed that if electrodes were placed at the center of posterior pathway, the posterior sensory and anterior motor pathways would be stimulated. However, if the current density produced at the posterior sensory pathway is larger than that at anterior motor pathway, the peak value would spread across the area of the posterior pathway from the lateral corticospinal tract to the fasciculus gracilis, as shown in Figure 1. If the electrodes were placed away from the posterior median line, such as above the left fasciculus cuneatus, the peak value would reach the opposite posterior horn nerve. Moreover, if the electrodes were placed at the posterior median line, the stimulated current density would be less when a stimulating current of $1 \mathrm{~mA}$ were to be applied, resulting in a current density of $0.008 \mathrm{~mA} / \mathrm{cm}^{2}$ at the anterior horn of spinal cord where $\theta$ is $180^{\circ}$ and $z=0, \rho=a$. When the stimulating current is increased to $5 \mathrm{~mA}$, the stimulated current density will increase correspondingly, with a current density of $0.025 \mathrm{~mA} / \mathrm{cm}^{2}$ at the anterior horn of the spinal cord where $\theta$ was approximately $180^{\circ}, Z=0, \rho=a$. These results suggest that increased stimulating current intensity will stimulate a whole transverse section of the spinal cord when electrodes are placed at the posterior median line. In clinical practices, intraoperative SCEP monitoring was performed with the electrode placed at the center of posterior pathway before surgical operation. During the surgical correction of spine deformity, the electrode may change the position from midline of the dorsal column to an oblique placement. It could decrease the stimulation to the spinal cord, leading to unexpected reduction in amplitude or absence of response and therefore to a false alarm to surgeons. Frequently, false warning of SCEP was found to result from the change of an electrode placement. Based on result of the present study, an increase of stimulation currency will achieve the same effect as baseline position, verifying 
whether the cause of abnormal changes in SCEP result from electrode position shift or neurological deficits. The understanding of electrical field and current density distribution under direct epidural electrical stimulation can help the monitoring specialists to avoid false monitoring outcomes.

In addition to SCEP monitoring, direct spinal cord stimulation was also used for pain management. In a previous study, Lee et at. studied the ability of dual parallel leads to steer stimulation between adjacent contacts on dual parallel leads for paresthesia-pain treatment by the finite element model of the low-thoracic spinal cord and its surrounding environment [16]. The study demonstrated that a multi-source system can target more central points of stimulation on the dorsal column than a single source system using finite element method, and multi-source system may allow for better optimization of paresthesia-pain overlap in patients to maximize coverage of painful areas[16]. Likewise, Gabriel et at. reported that the electric fields generated by three disc electrodes electrodes located epidurally were about three times more intense than that placed outside the spine, and uniformly distributed electric fields were obtained with five disc electrodes placed around the dura mater[17]. Results from the present mathematical model and simulation study were supported by findings from previous studies [16-17]. Moreover, this paper presented quantitative results with three-dimensional potential/current distribution within dura matter and the cord, as well as the contour map with different $\rho$ within spinal cord.

\section{CONCLUSIONS}

Due to the small electrical conductivity of dura matter tissues, the conduction of electrical stimulation is narrow and the stimulated current field primarily distributes towards the transverse section of the spinal cord. The effect of direct epidural electrical stimulation is mainly on local tissue surrounding the electrodes, which concentrates around two simulated positions. This suggests that the pair of electrodes should be transversely placed by at least the stimulated sections. The above figures demonstrate that potential fields and current densities in each tissue in this model are not single valued, but rather extend a range of magnitudes. The current density in the 
outer spinal cord was greater than the inner region. In addition, low stimulating intensity can excite white matter only on the surface conduction tracts like spinocerebellar tracts. If the stimulation is expected to reach deeper tractslike the lateral corticospinal tract and anterior corticospinal tract, or even deeper in reticulospinal tracts, the stimulating intensity should be increased. 


\section{Reference}

[1] J.J. Struijk, J. Holsheimer, H.B. Boom, Excitation of dorsal root fibers in spinal cord stimulation: a theoretical study, IEEE transactions on bio-medical engineering, 40 (1993) 632-639.

[2] J.J. Struijk, J. Holsheimer, B.K. van Veen, H.B. Boom, Epidural spinal cord stimulation: calculation of field potentials with special reference to dorsal column nerve fibers, IEEE transactions on bio-medical engineering, 38 (1991) 104-110.

[3] M.M. Pinter, F. Gerstenbrand, M.R. Dimitrijevic, Epidural electrical stimulation of posterior structures of the human lumbosacral cord: 3. Control Of spasticity, Spinal cord, 38 (2000) 524-531.

[4] F. Rattay, K. Minassian, M.R. Dimitrijevic, Epidural electrical stimulation of posterior structures of the human lumbosacral cord: 2. quantitative analysis by computer modeling, Spinal cord, 38 (2000) 473-489.

[5] C.H. Tator, K. Minassian, V.K. Mushahwar, Spinal cord stimulation: therapeutic benefits and movement generation after spinal cord injury, Handbook of clinical neurology, 109 (2012) 283-296.

[6] E.A. Grossi, J.C. Laschinger, K.H. Krieger, I.M. Nathan, S.B. Colvin, M.R. Weiss, F.G. Baumann, Epidural-evoked potentials: a more specific indicator of spinal cord ischemia, The Journal of surgical research, 44 (1988) 224-228.

[7] T.R. Moshonkina, A.N. Makarovski, I.N. Bogacheva, N.A. Scherbakova, A.A. Savohin, Y.P. Gerasimenko, Effects of spinal cord electrical stimulation in patients with vertebrospinal pathology, Bulletin of experimental biology and medicine, 153 (2012) 16-20.

[8] S. Harkema, Y. Gerasimenko, J. Hodes, J. Burdick, C. Angeli, Y. Chen, C. Ferreira, A. Willhite, E. Rejc, R.G. Grossman, V.R. Edgerton, Effect of epidural stimulation of the lumbosacral spinal cord on voluntary movement, standing, and assisted stepping after motor complete paraplegia: a case study, Lancet, 377 (2011) 1938-1947.

[9] K. Minassian, U. Hofstoetter, K. Tansey, W. Mayr, Neuromodulation of lower limb motor control in restorative neurology, Clinical neurology and neurosurgery, 114 (2012) 489-497.

[10] K.W. Altman, R. Plonsey, Point source nerve bundle stimulation: effects of fiber diameter and depth on simulated excitation, IEEE transactions on bio-medical engineering, 37 (1990) 688-698.

[11] E.L. Carter, Jr., S.R. Pollack, C.T. Brighton, Theoretical determination of the current density distributions in human vertebral bodies during electrical stimulation, IEEE transactions on bio-medical 
engineering, 37 (1990) 606-614.

[12] D. Panescu, J.G. Webster, R.A. Stratbucker, A nonlinear finite element model of the electrode-electrolyte-skin system, IEEE transactions on bio-medical engineering, 41 (1994) 681-687.

[13] J.T. Rubinstein, Axon termination conditions for electrical stimulation, IEEE transactions on bio-medical engineering, 40 (1993) 654-663.

[14] J.J. Struijk, J. Holsheimer, G.H. Spincemaille, F.L. Gielen, R. Hoekema, Theoretical performance and clinical evaluation of transverse tripolar spinal cord stimulation, IEEE transactions on rehabilitation engineering : a publication of the IEEE Engineering in Medicine and Biology Society, 6 (1998) 277-285.

[15] J.J. Struijk, J. Holsheimer, G.G. van der Heide, H.B. Boom, Recruitment of dorsal column fibers in spinal cord stimulation: influence of collateral branching, IEEE transactions on bio-medical engineering, 39 (1992) 903-912.

[16] D. Lee, E. Gillespie, K. Bradley, Dorsal Column Steerability with Dual Parallel Leads using Dedicated Power Sources: A Computational Model, J Vis Exp, 48 (2011) 2443.

[17] Hernandez-Labrado GR, Polo JL, Lopez-Dolado E, et al, Spinal cord direct current stimulation: finite element analysis of the electric field and current density: Med Biol Eng Comput, 2011, 49(4) $17-29$.

[18] T. GJ, Principles of Anatomy and Physiology, 7th Edition ed., IMPRINT New York: HarperCollins, 1996.

[19] W.G.N. Whittaker E. T. , A Course of Modern Analysis, Cambridge University Press, 2002.

[20] E.L. Carter, Jr., E.J. Vresilovic, S.R. Pollack, C.T. Brighton, Field distributions in vertebral bodies of the rat during electrical stimulation: a parametric study, IEEE transactions on bio-medical engineering, 36 (1989) 333-345.

[21] J.L. Parker, D.M. Karantonis, P.S. Single, M. Obradovic, M.J. Cousins, Compound action potentials recorded in the human spinal cord during neurostimulation for pain relief, Pain, 153 (2012) 593-601. 


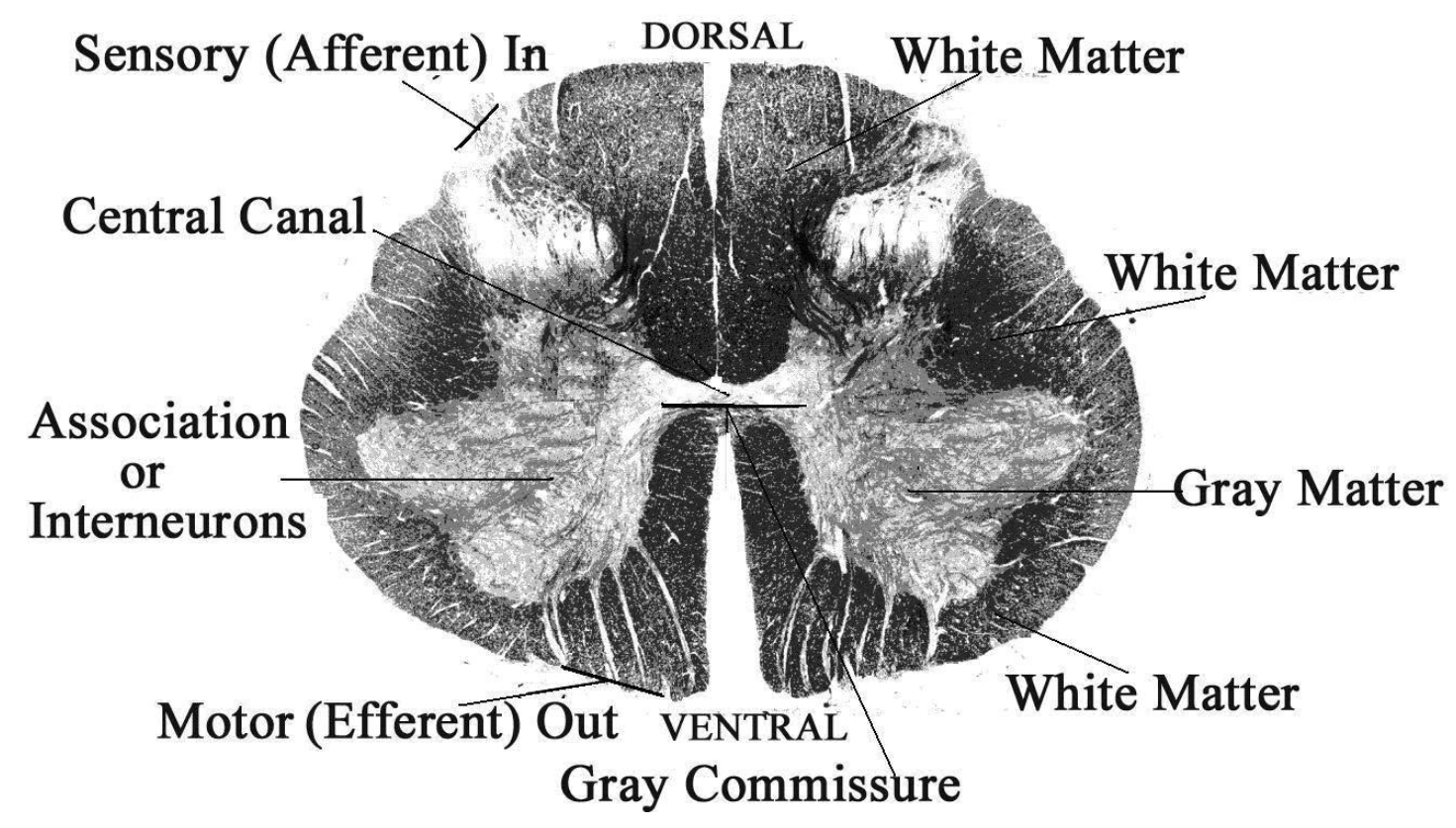

Fig.1 Transverse section of spinal cord 


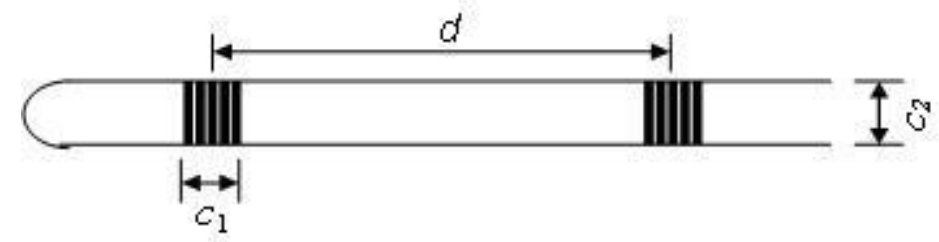

Fig.2 Stimulating electrode 


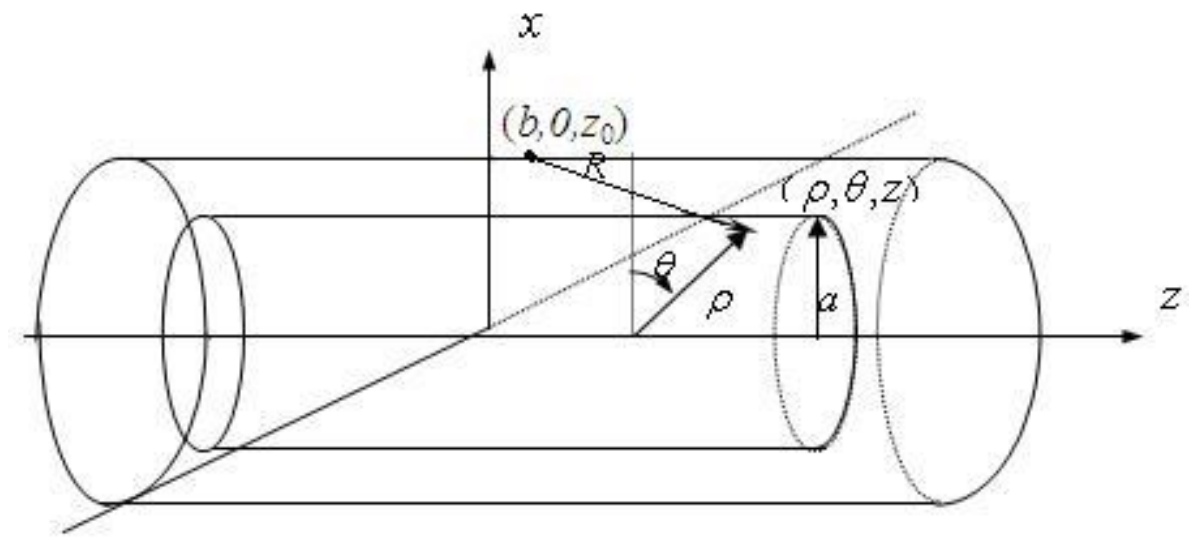

Fig.3 Spinal cord with dura matter stimulated by point electrical source 


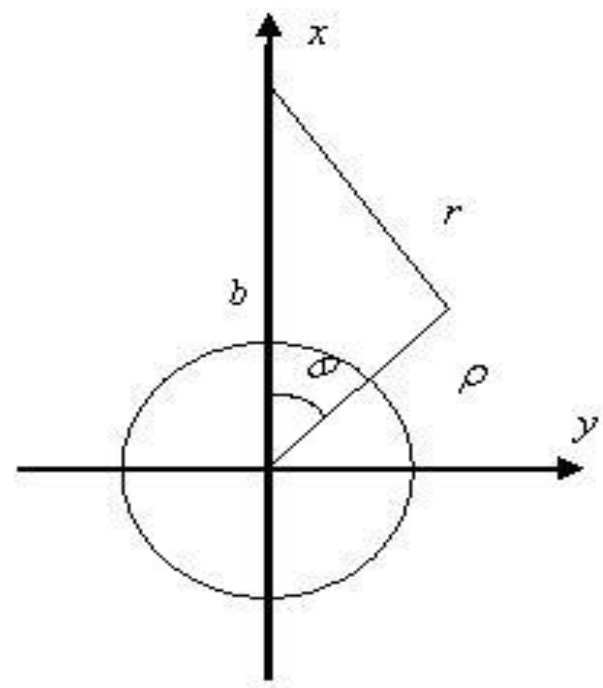

Fig.4 Projection to the $x y$ coordinate plane 


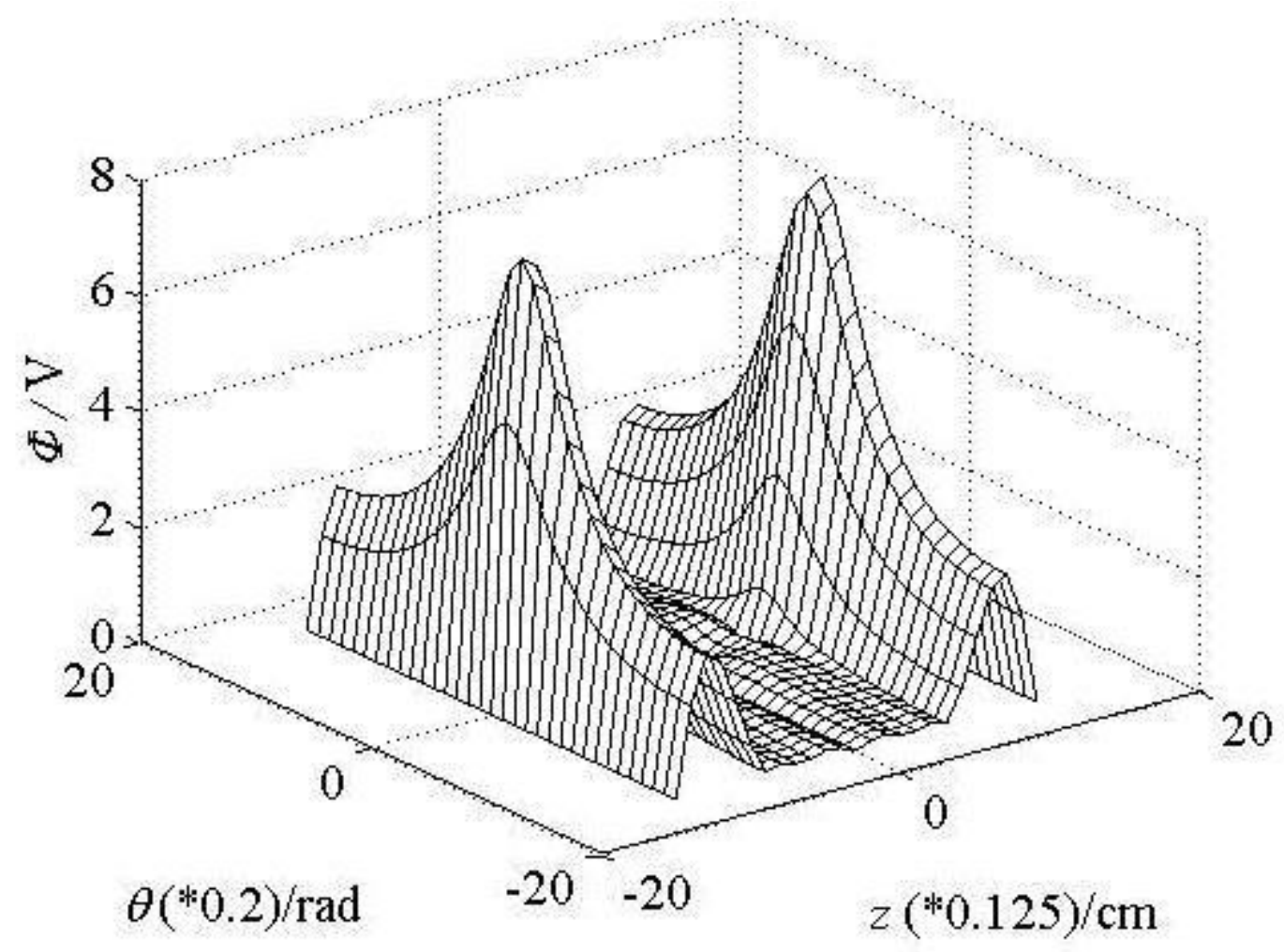

(a)

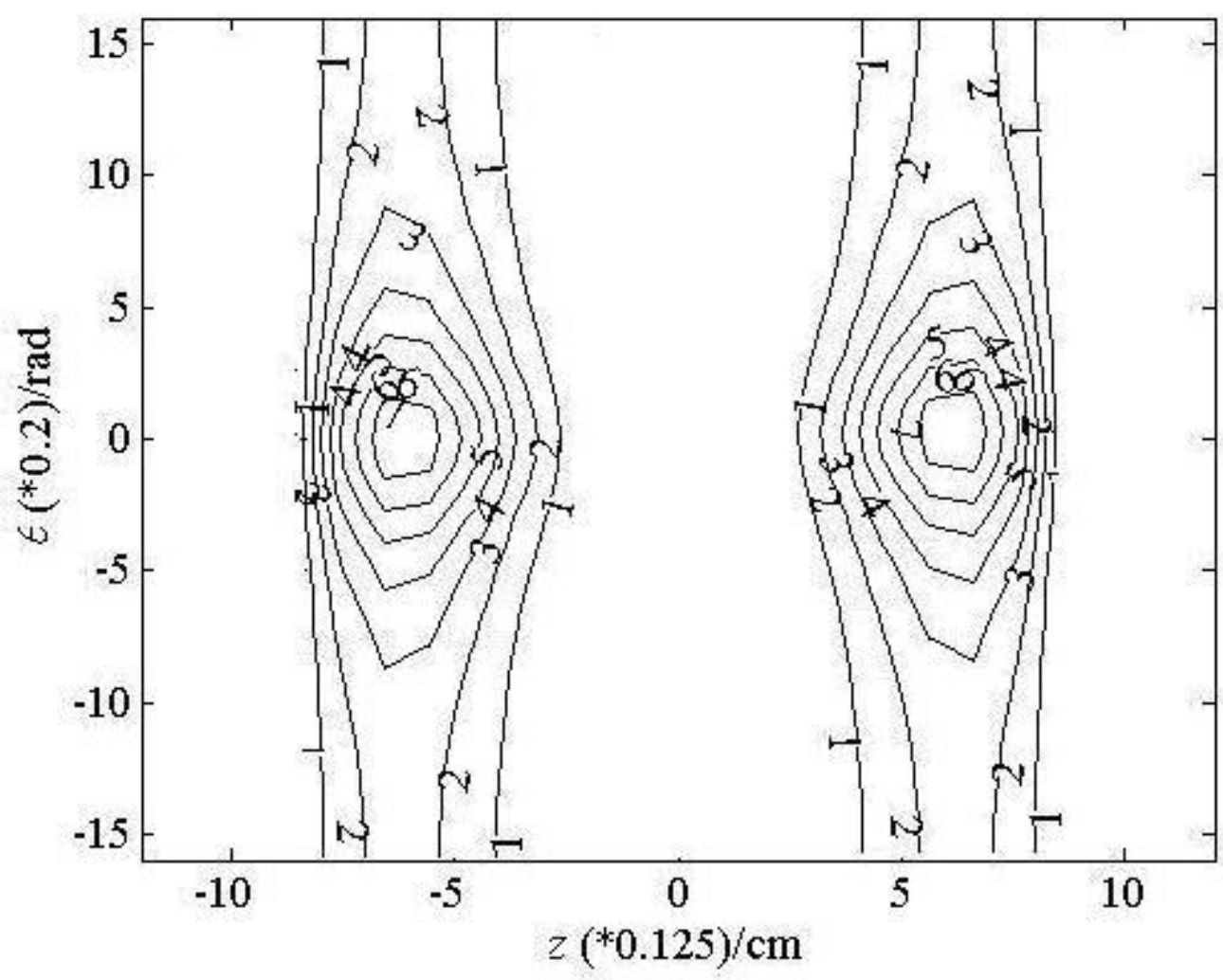

(b)

Fig.5 Potential distribution within dura matter.

(a) the three-dimensional map; (b)the contour map 


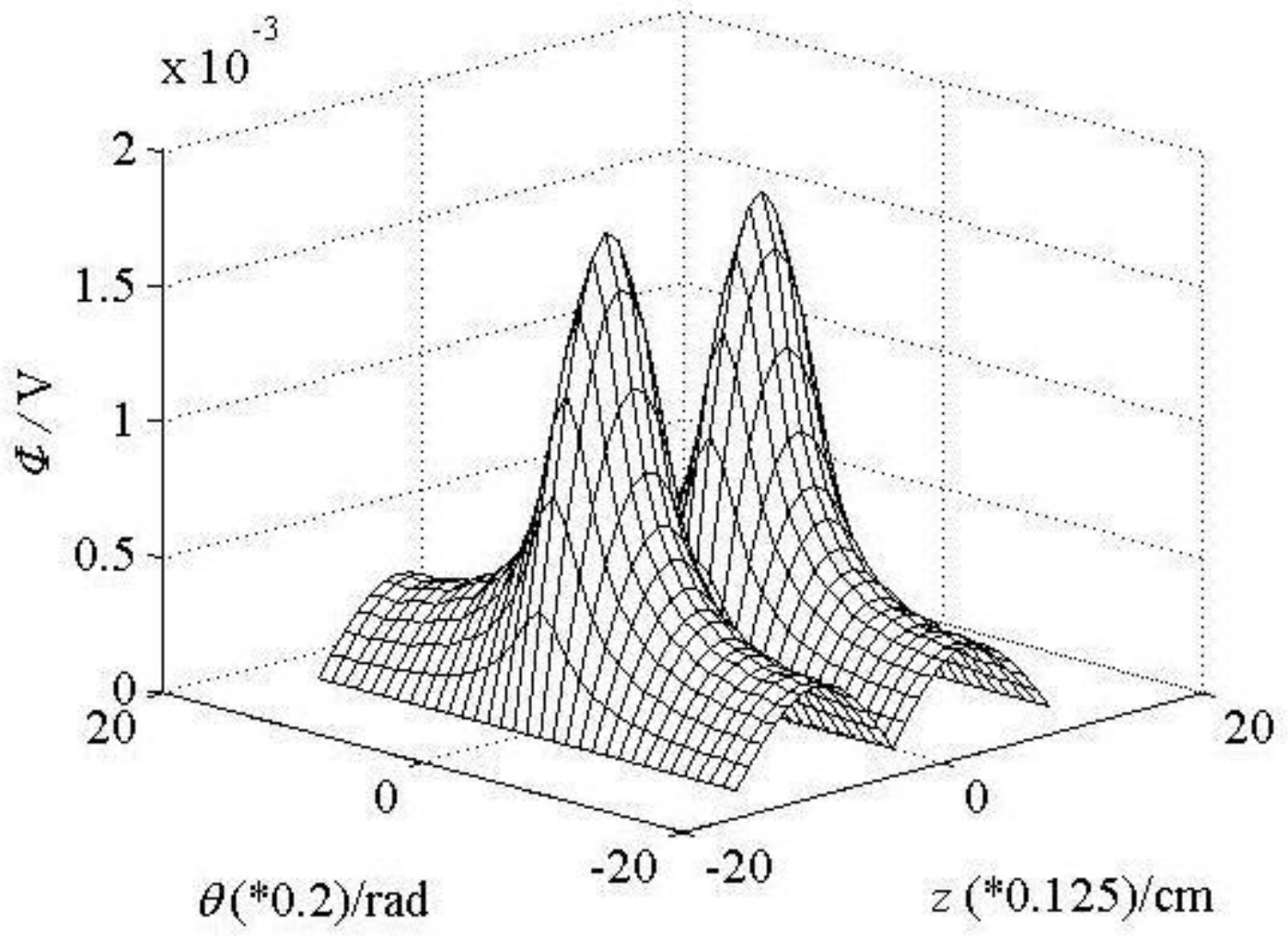

(a)

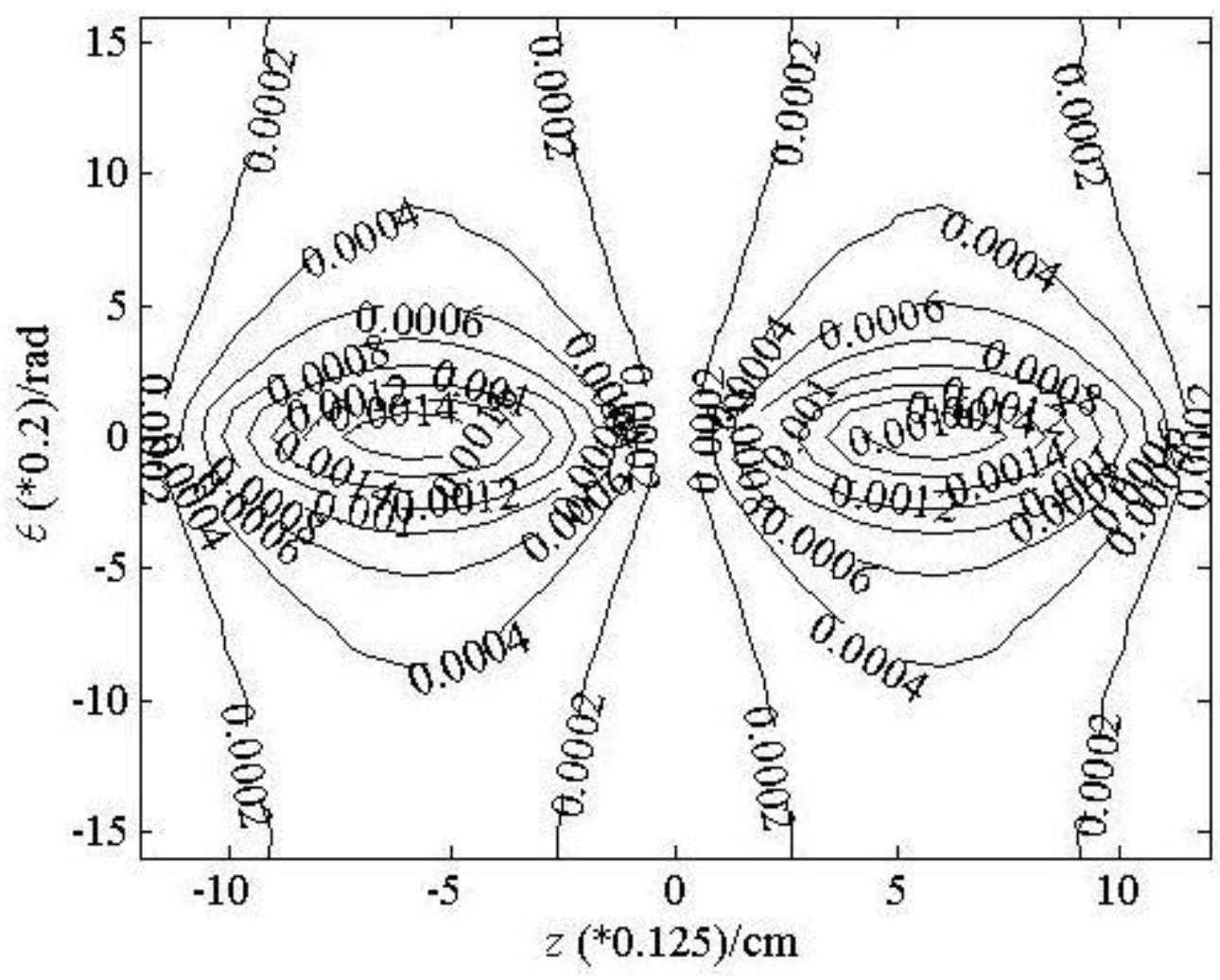

(b)

Fig.6 Potential distribution within spinal cord when $\rho=0.5 \mathrm{~cm}$.

(a) the three-dimensional map; (b)the contour map 


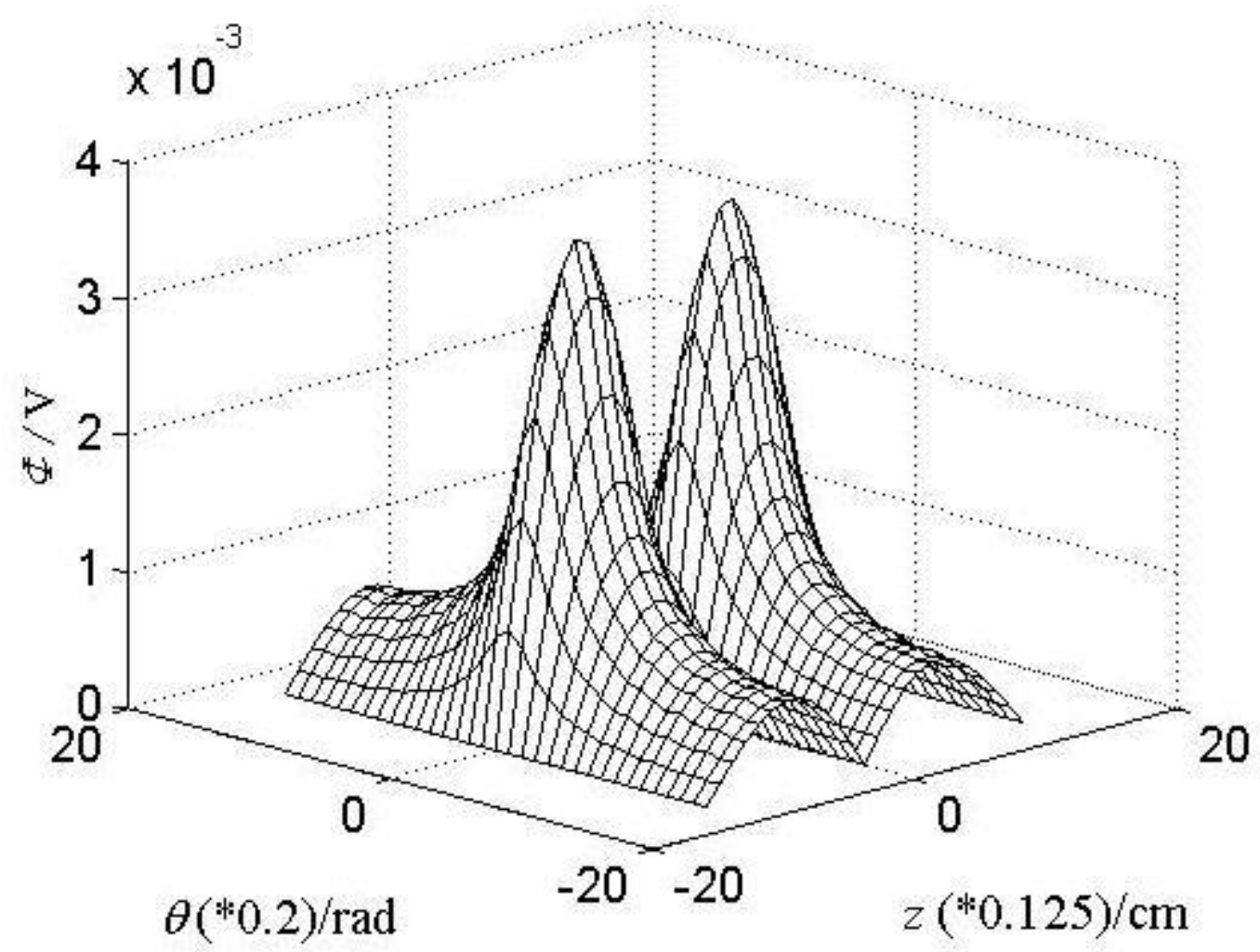

(a)

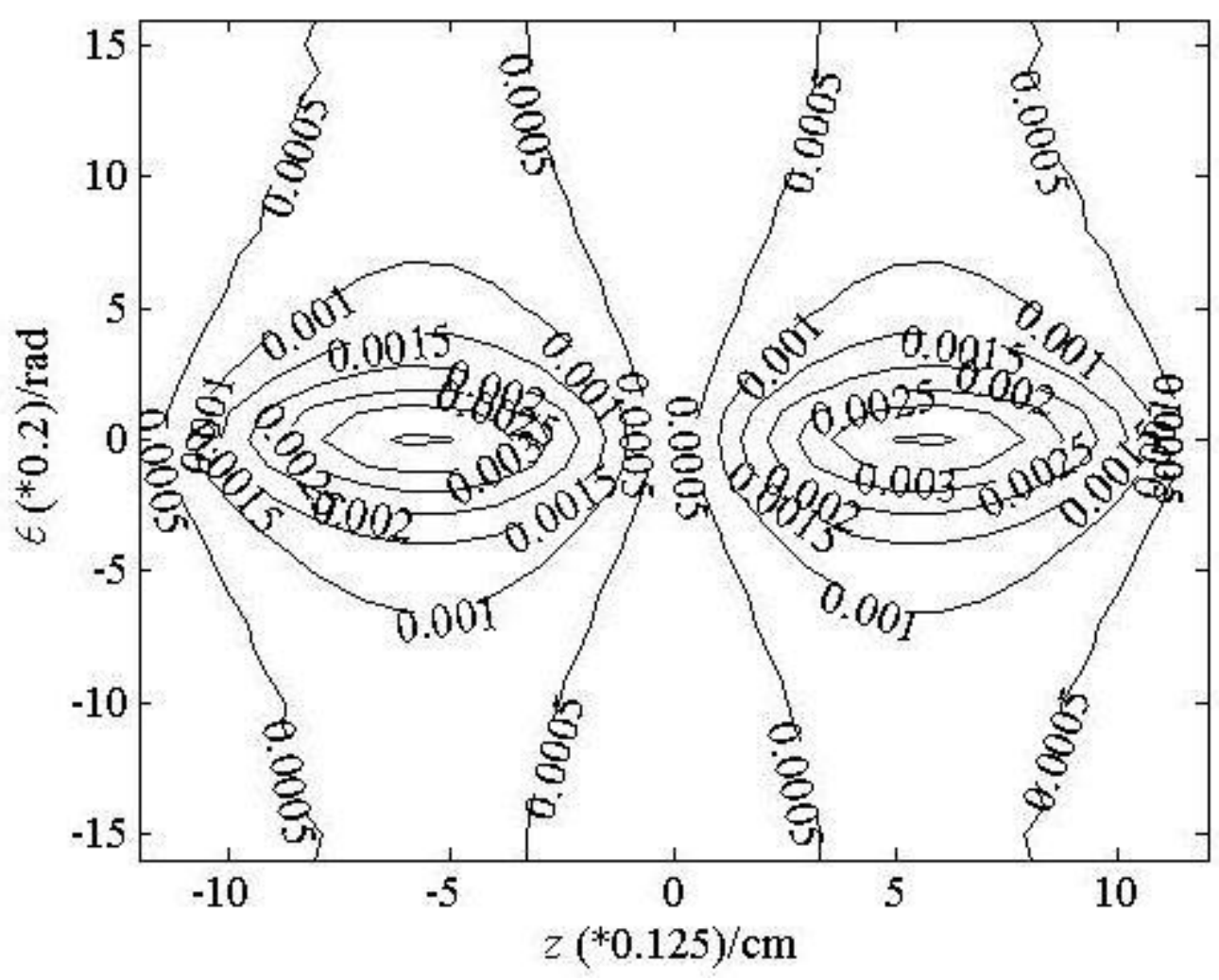

(b)

Fig.7 Potential distribution within spinal cord when $\rho=1.0 \mathrm{~cm}$.

(a) the three-dimensional map; (b)the contour map 


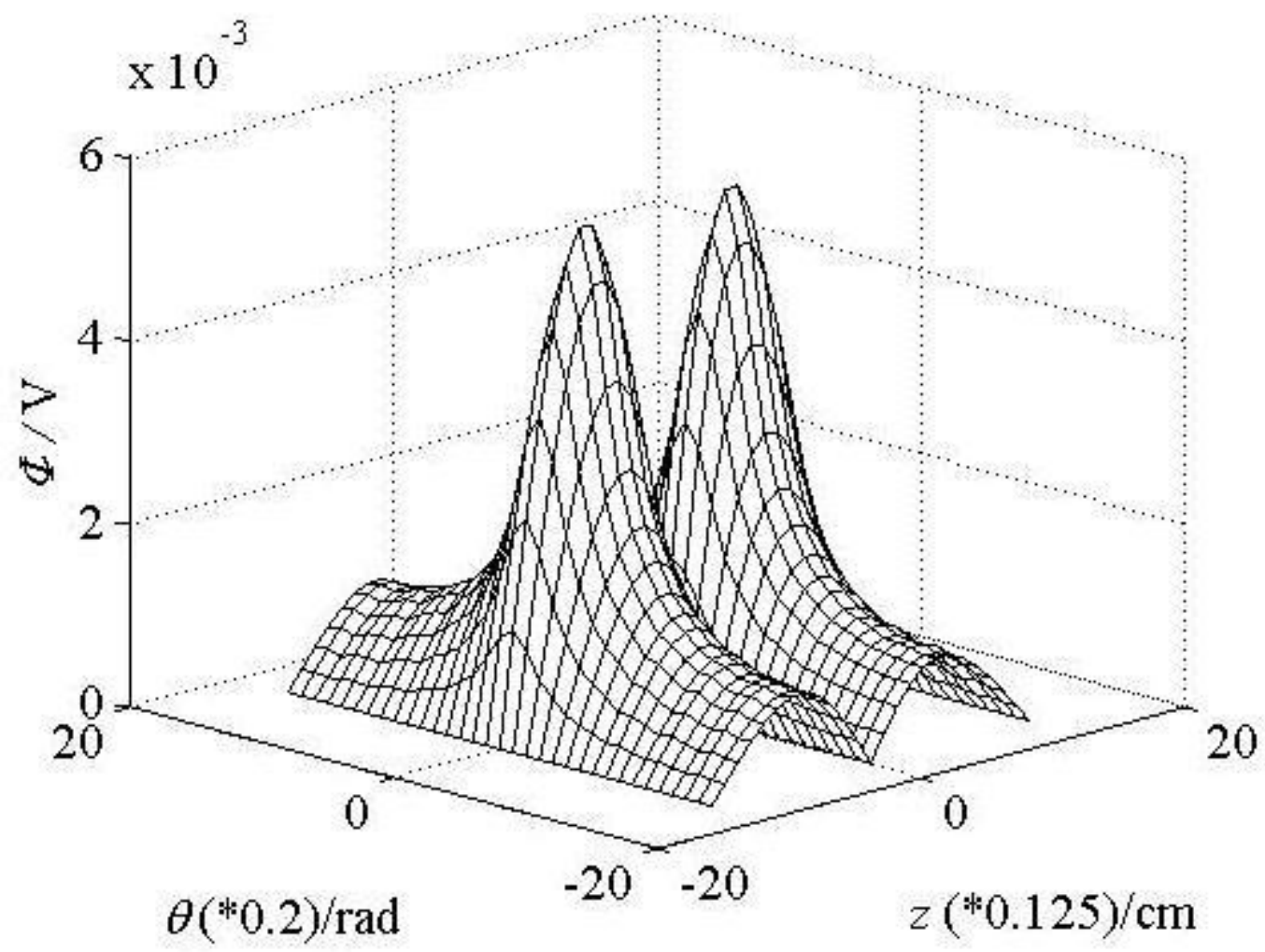

(a)

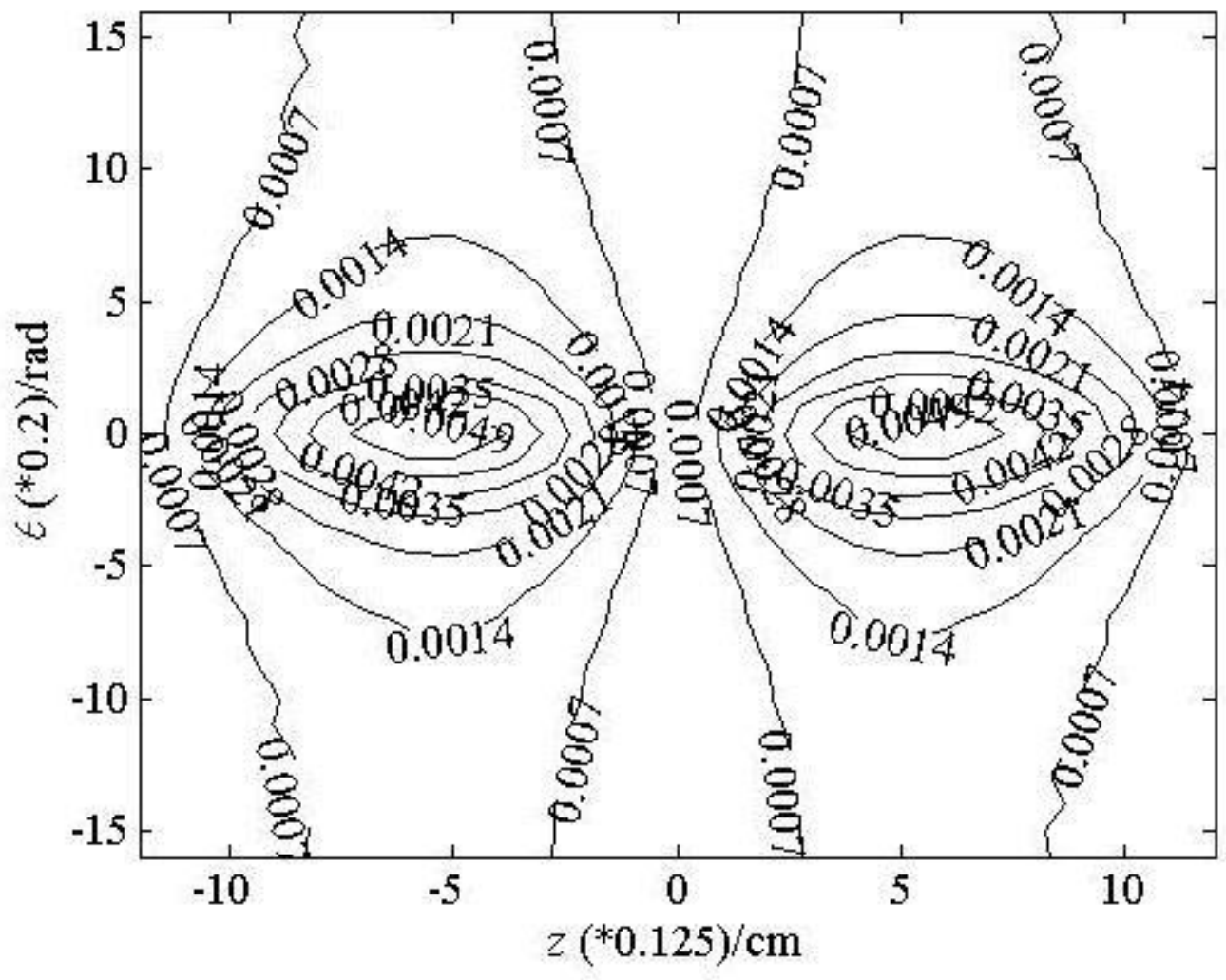

(b)

Fig.8 Potential distribution within spinal cord when $\rho=1.5 \mathrm{~cm}$.

(a) the three-dimensional map; (b)the contour map 


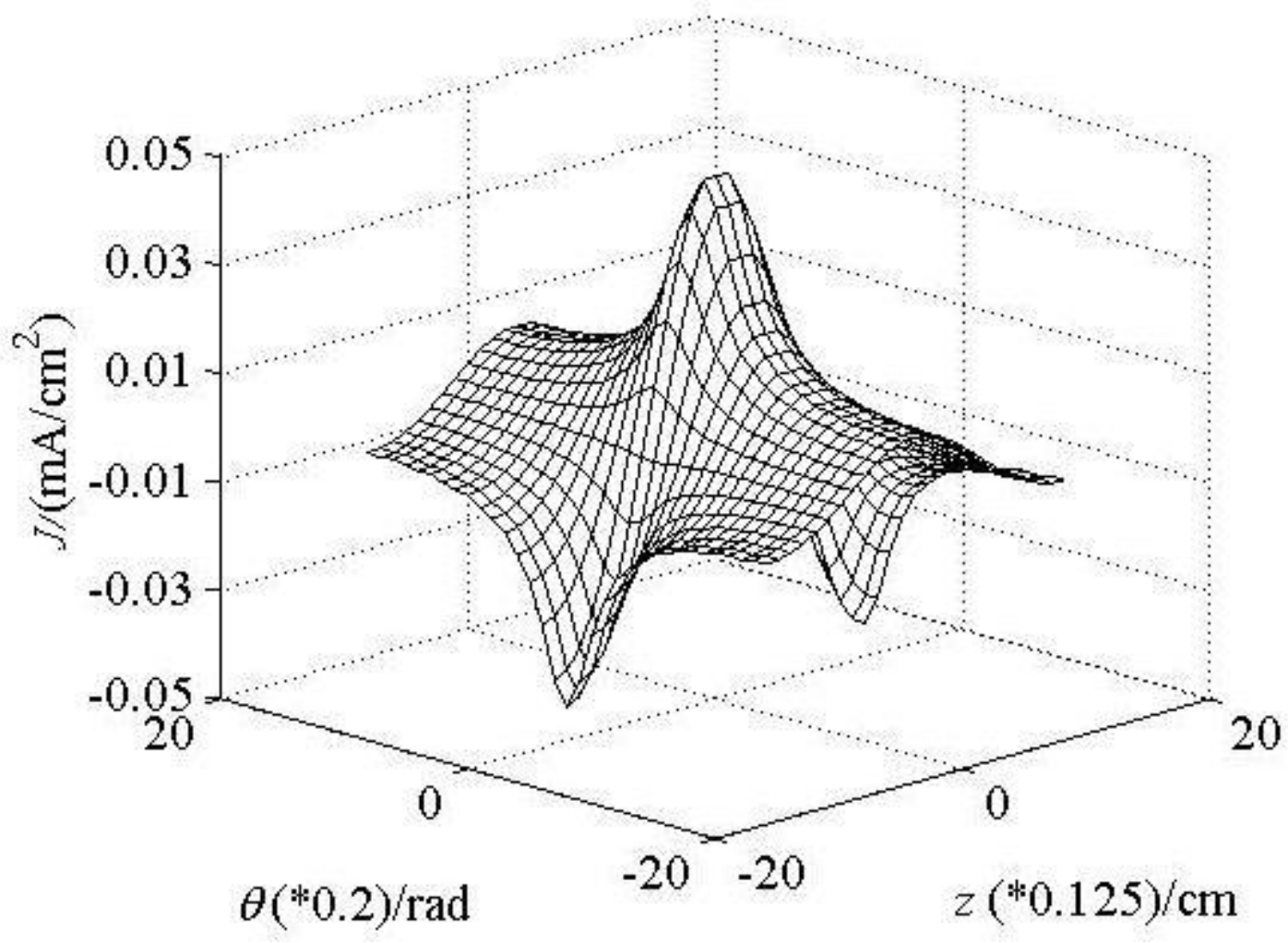

(a)

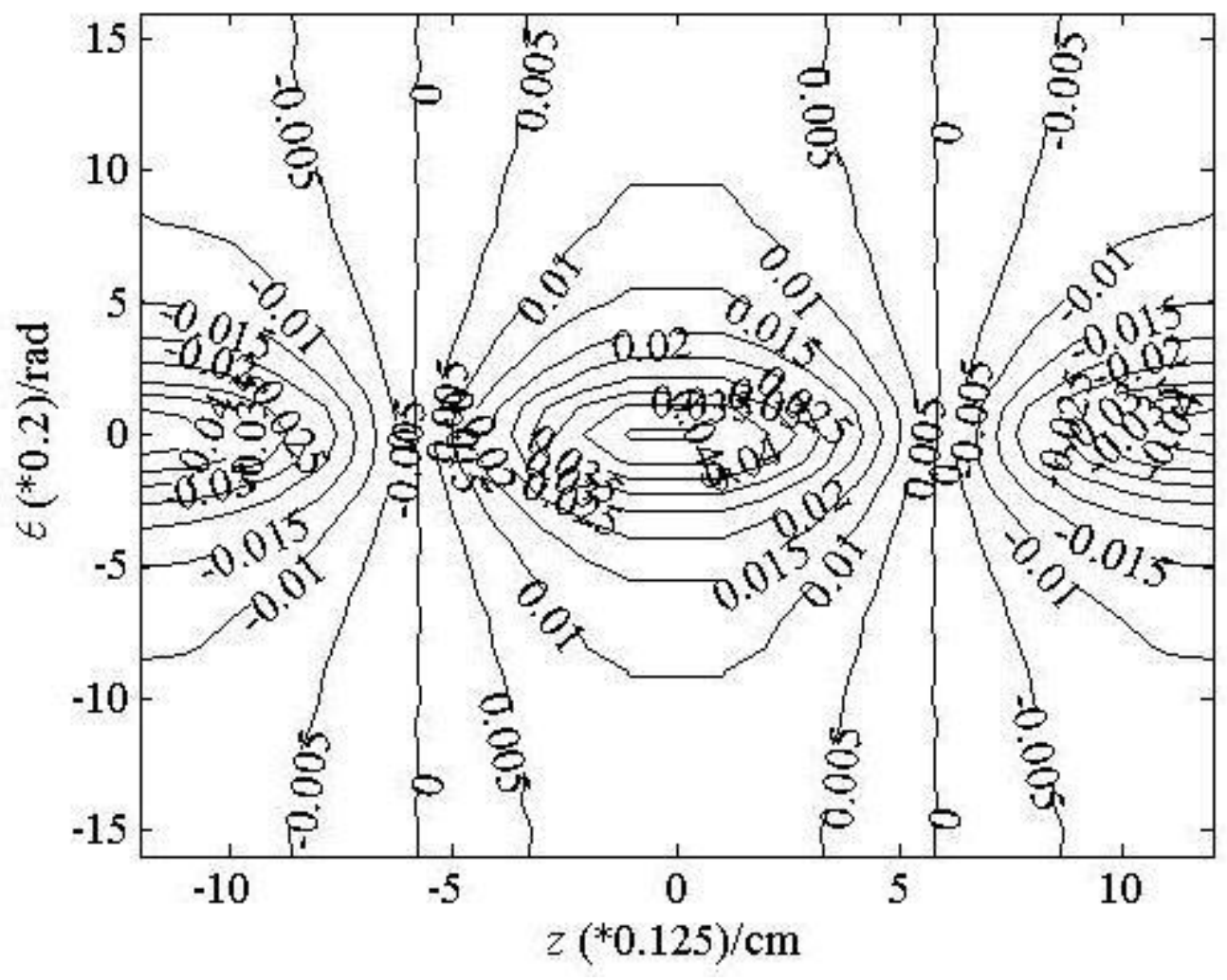

(b)

Fig.9 Current distribution within spinal cord when $\rho=0.5 \mathrm{~cm}$.

(a) the three-dimensional map; (b)the contour map 


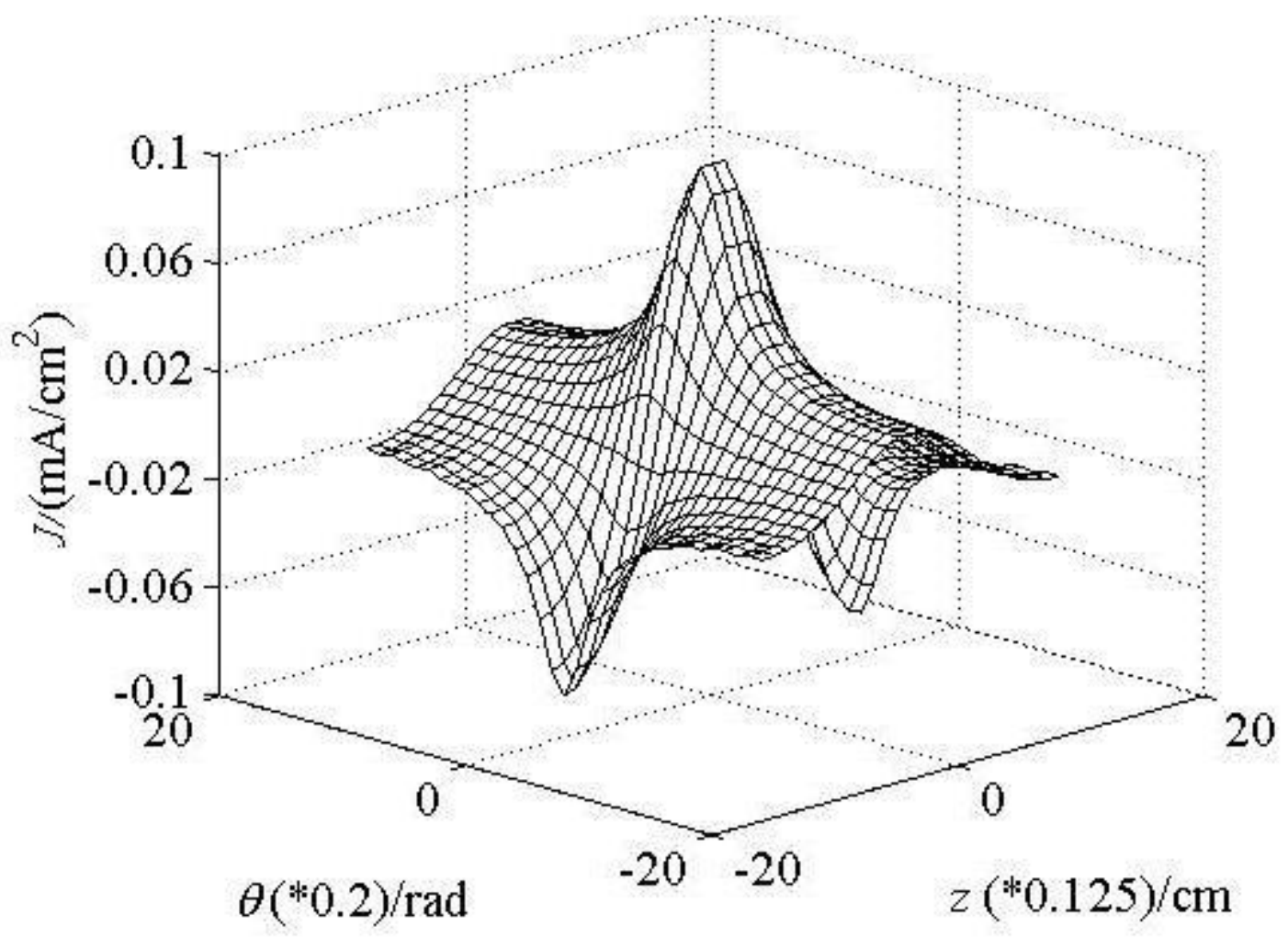

(a)

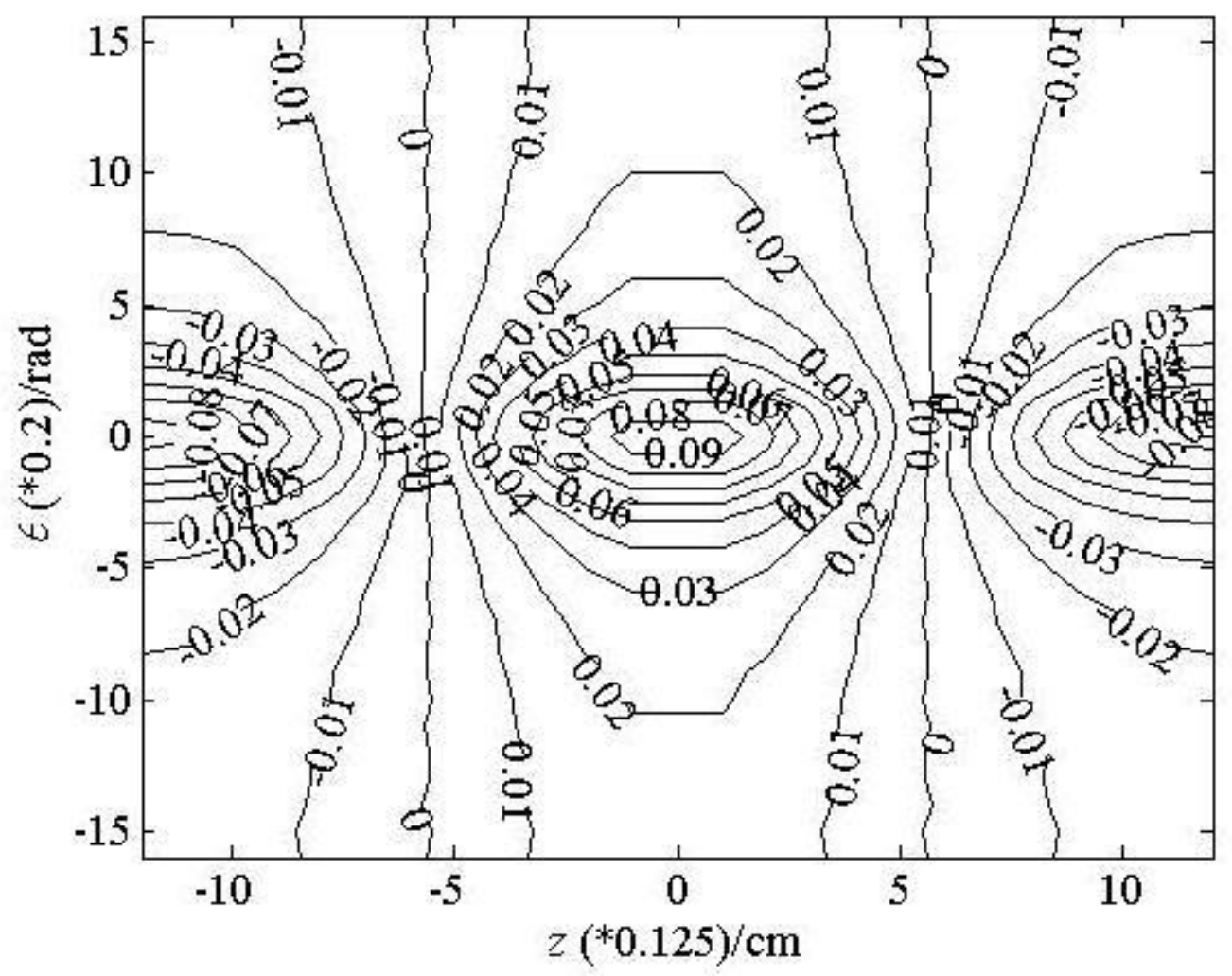

(b)

Fig.10 Current distribution within spinal cord when $\rho=1.0 \mathrm{~cm}$.

(a) the three-dimensional map; (b)the contour map 


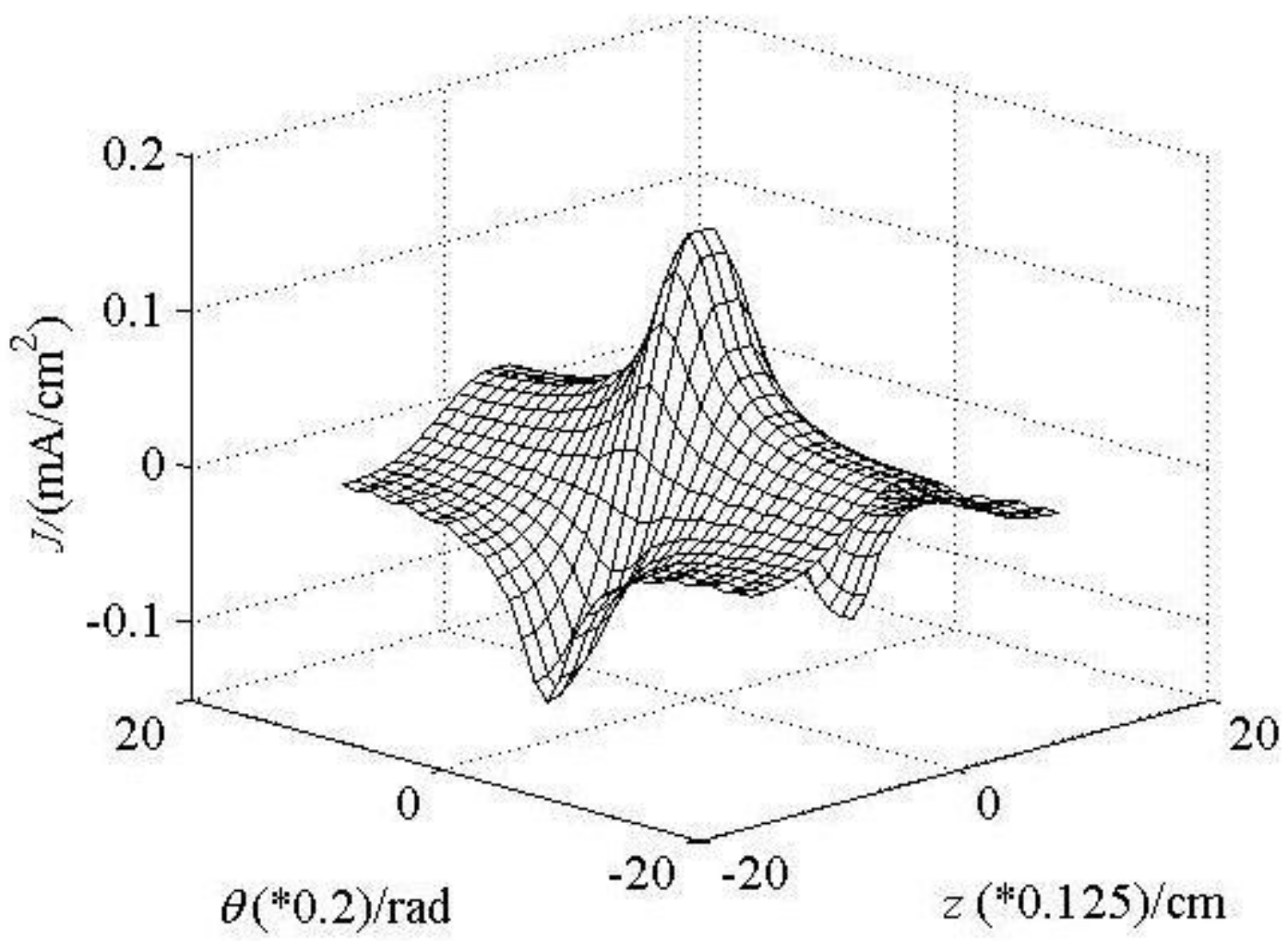

(a)

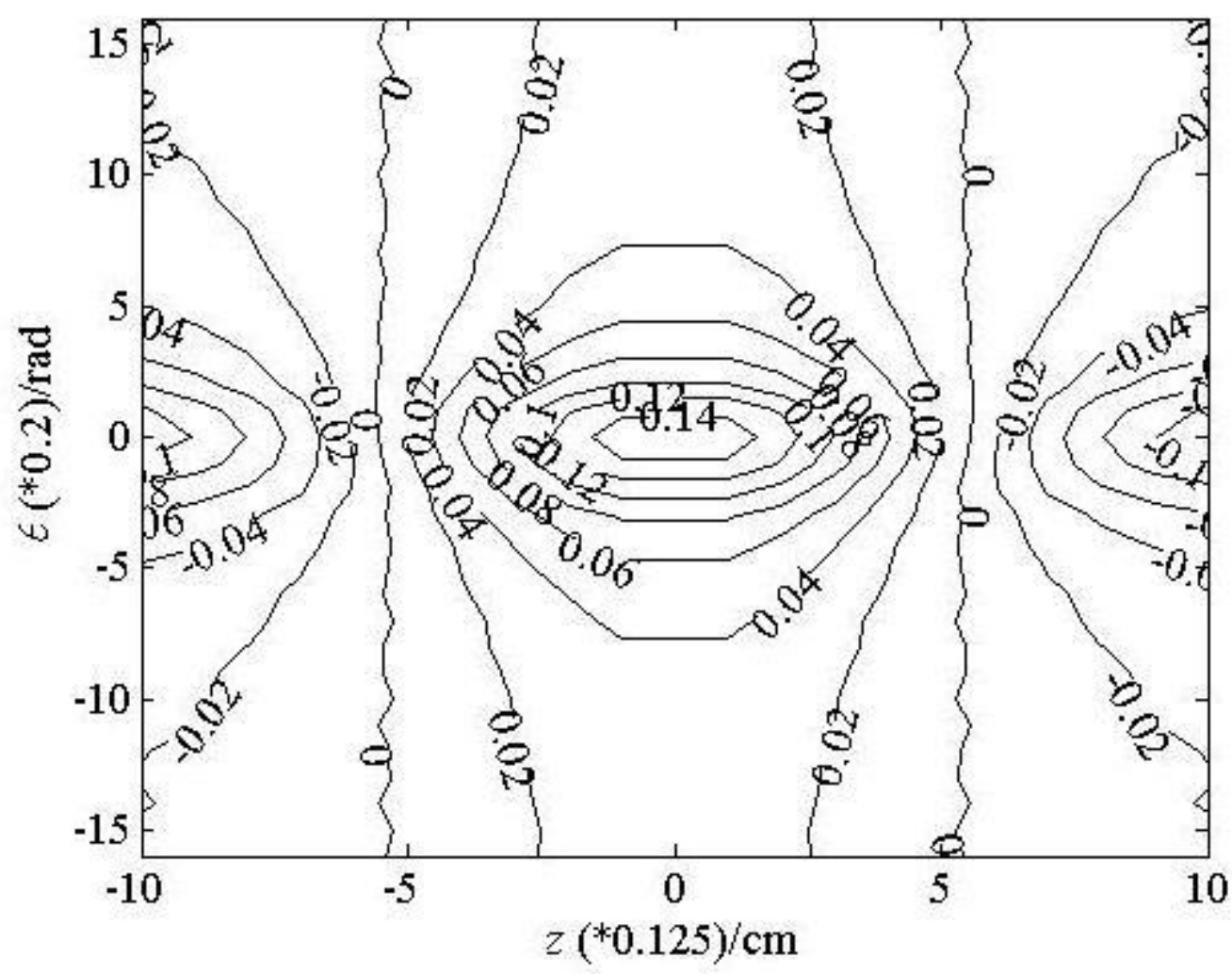

(b)

Fig.11 Current distribution within spinal cord when $\rho=1.5 \mathrm{~cm}$.

(a) the three-dimensional map; (b)the contour map 